\title{
Erythema and induration of the Bacillus Calmette-Guérin site for diagnosing Kawasaki disease
}

\author{
Ann Chuan En Loh $\underline{h}^{1}$, MBBs, Phek Hui Jade $\underline{K u a}^{2}$, MBBS, MrCs, Ze Lei $\underline{T a n}^{3}$, MBBS
}

INTRODUCTION Kawasaki disease (KD) is a challenging diagnosis. Erythema and induration of the Bacillus Calmette-Guérin (BCG) site is increasingly recognised as a significant clinical clue. However, there is little data to support its specificity for $\mathrm{KD}$ as compared to other febrile illnesses. We aimed to evaluate BCG reaction or induration as a diagnostic tool for KD. METHODS A retrospective case-controlled study of patients discharged with a diagnosis of KD from 2007 to 2010 was conducted. Another group of patients admitted over the same period for possible KD, but later found not to have KD, served as control.

RESULTS Significantly more infants with KD (69.7\%) had BCG site changes than older children $(27.8 \% ; p<0.001)$. It also presented earlier in the course of $\mathrm{KD} ;<5$ days $(53.3 \%)$ compared to $\geq 5$ days of fever $(30.0 \% ; \mathrm{p}<0.001)$. Positive predictive value of BCG site reaction or induration for KD was 90.8\% (95\% confidence interval [ $\mathrm{Cl}$ ] 0.819-0.962) for infants and $96.2 \%(95 \% \mathrm{Cl} 0.868-0.995)$ for older children. The prevalence rate of changes at the BCG site was $9.9 \%$ among patients with non-KD febrile illnesses and $42.6 \%$ among patients with KD.

CONCLUSION BCG site reaction or induration is a useful clinical clue for the diagnosis of KD in both infants and older children, with a higher prevalence in infants. Physicians should consider KD in children with febrile illness and redness or crust formation at the BCG site, especially in view of low rates of BCG reaction or induration in non-KD febrile illnesses.

Keywords: BCG inoculation site, BCG reactivation, Kawasaki disease

\section{INTRODUCTION}

Kawasaki disease (KD) is one of the most common vasculitides of childhood, primarily affecting children younger than five years of age. (1) Prompt recognition and treatment is crucial due to the time-sensitive risk of developing cardiac sequelae, such as coronary aneurysms in up to $15 \%-25 \%$ of untreated children. ${ }^{(2)}$ This may further result in myocardial infarction, ischaemic heart disease or sudden death. ${ }^{(3,4)}$

$\mathrm{KD}$ is a clinical diagnosis. In the American Heart Association (AHA) scientific statement (2004), this was defined as the presence of $\geq 5$ days of fever and $\geq 4$ of five principle clinical features (i.e. bilateral nonexudative conjunctivitis, erythema of the lips and oral mucosa, changes in the extremities, rash and cervical lymphadenopathy). ${ }^{(5)}$ Alternatively, patients with $\geq 5$ days of fever and $<4$ of the aforementioned symptoms can be diagnosed when coronary artery disease is detected by twodimensional echocardiography or coronary angiography. The AHA also advises that the diagnosis can be made in the presence of $\geq 4$ principle criteria, and should be strongly considered in a young child with unexplained fever for $\geq 5$ days and any of the principle clinical features. The diagnosis of KD can only be made on exclusion of other diagnoses.

The AHA guidelines recognise that not all clinical features present simultaneously and watchful waiting is often necessary before a diagnosis of KD can be made. ${ }^{(5)}$ Currently, two diagnostic guidelines exist for this entity known as incomplete KD. According to Ayusawa et $\mathrm{al}^{\left({ }^{(6)}\right.}$ a diagnosis of incomplete $\mathrm{KD}$ is made with four principle signs and the presence of coronary artery aneurysm. A Committee of Experts under the AHA proposed for incomplete KD to be considered in children with unexplained fever for $\geq 5$ days associated with two or three of the principle features in the AHA criteria. They also advised a high index of suspicion for KD in infants aged less than six months presenting with unexplained fever of $\geq 7$ days, with laboratory evidence of systemic inflammation. ${ }^{(5)}$

Both guidelines recognise erythema and induration at the Bacillus Calmette-Guérin (BCG) inoculation site to be a significant non-cardiac feature of $\mathrm{KD} .{ }^{(5,6)}$ It was first highlighted in the Japanese literature as a specific and early sign of $\mathrm{KD},{ }^{(7)}$ and has been well established as a useful tool for enhancing the diagnosis of incomplete $\mathrm{KD}$, predominantly in countries with a national BCG vaccination programme. ${ }^{(8-10)}$

A recent Taiwanese study in 2016 characterised and graded the reaction at the BCG site in KD via dermatoscopy. ${ }^{(11)}$ It identified bull's-eye pattern under dermatoscopy as a good clinical clue for systemic involvement, i.e. abnormal elevation of liver enzymes and cardiac complications (dilatation of the coronary arteries). However, there remains a distinct lack of established data detailing the specificity of changes at the BCG site to KD and its significance in evaluating the severity of disease.

Through our study, we hoped to evaluate the specificity of erythema and induration at the BCG inoculation site for the diagnosis of KD and to quantify the presence of this BCG reaction in KD and other febrile illnesses.

This research project was approved by the cluster institution review board with waiver of consent.

${ }^{1}$ Department of General Paediatrics, ${ }^{2}$ Department of Children's Emergency, KK Women's and Children's Hospital, ${ }^{3}$ Department of General Paediatrics, Khoo Teck Puat-National University Children's Medical Institute, National University Hospital, Singapore

Correspondence: DrAnn Loh, Medical Officer, Department of Paediatrics, KK Women's and Children's Hospital, 100 Bukit Timah Road, Singapore 229899. ann.loh@mohh.com.sg 


\section{METHODS}

We conducted a retrospective case-control study involving patients admitted to KK Women's and Children's Hospital, Singapore, from the children's emergency between April 2007 and December 2010, who were then discharged from the ward with a diagnosis of KD. Control patients, or patients with non-KD febrile illnesses, were a separate group of patients admitted from the children's emergency with suspected KD during the same period, who were later discharged from the ward with a final diagnosis that was not KD.

Patients were assessed by emergency physicians in the children's emergency and re-evaluated by paediatricians in the ward. In the children's emergency, suspicion of KD was based on the constellation of clinical features present at the time of admission. In the ward, diagnosis by paediatricians was made based on the presence of the AHA clinical criteria or other features described in the literature, response to treatment with intravenous immunoglobulin or presence of suggestive echocardiography findings. The diagnosis of incomplete or atypical KD was made if the patient did not fully fulfil the AHA criteria for KD, but was treated as KD with intravenous immunoglobulin (based on the ward paediatrician's clinical decision) and showed response to treatment. The absence of KD in the control group was determined by the ward physician through the evolution of clinical features that later were not suggestive of KD or the response to other treatment (e.g. symptomatic treatment or antibiotics).

Following identification of patients, data was abstracted for the presence of the AHA clinical features, other suggestive features that have been described in the literature (e.g. erythema and induration of BCG inoculation site, irritability) and twodimensional echocardiography findings (if performed as inpatient).

Patients were identified through the Outpatient Electronic Clinical system and data was collected simultaneously by two co-authors based on a pre-established variable definition glossary to ensure accuracy of data abstraction. Data was de-identified (patient name and National Registration Identity Number replaced with patient number) before being released to the biostatistician for analysis.

Statistical analyses were performed using SAS 9.2 (SASC, Carey, NC, USA). Statistical significance was set at $\mathrm{p}<0.05$. Wilcoxon rank-sum tests were used to compare continuous variables between the two patient groups. Chi-square and Fisher's exact tests were used to compare proportions, where appropriate.

For demographic data, age, gender and ethnicity were summarised and presented as mean \pm standard deviation or count (\%) by KD status (overall, true KD and control) and age (overall, infants [age $<1$ year] and older children [age $\geq 1$ year]), and compared between KD and control groups by age group.

Further analysis was performed for significant epidemiological characteristics of patients with KD with erythema and induration of the BCG inoculation site in the ward according to age, gender, ethnicity, duration of fever and presence of other classic features of KD. The prevalence of the aforementioned characteristics was presented as count $(\%)$, and then compared between patients with erythema and induration of the BCG inoculation site and those without.

\section{RESULTS}

In total, 661 patients were deemed eligible based on the above criteria and included in the analysis. Among these, 420 (63.5\%) patients had KD, while 241 (36.5\%) were patients with non-KD febrile illnesses or controls. There was a preponderance of male $(62.6 \%)$ patients. Ethnically, Chinese (77.3\%) patients comprised the highest proportion, followed by patients of Malay $(12.3 \%)$, and Indian and other ethnicities (10.5\%). The overall mean age was $2.2 \pm 2.4$ years while median age was 1 (interquartile range [IQR] 0-3) years. Both patient and control groups had similar demographics (Table I).

A discharge diagnosis of KD was seen for 139/176 (79.0\%) infants and 281/485 (57.9\%) older children. The mean age of older children with KD was significantly lower than that of control patients $(2.4 \pm 1.7$ years vs. $3.8 \pm 2.7$ years; $p<0.0001)$.

In the ward, $121 / 176(68.8 \%)$ infants and 249/485 (51.3\%) older children were checked for erythema and induration of the BCG inoculation site (Table II). Overall, 279/420 (66.4\%) patients with $\mathrm{KD}$ were checked for BCG site reaction or induration. Among the 279 patients with KD who were checked, 119 (42.7\%) patients had BCG reaction or induration. Meanwhile, 91/241 (37.8\%) patients with non-KD diagnoses were checked for BCG site changes, out of which $9(9.9 \%)$ patients were found to have erythema or induration of the BCG inoculation site (Fig. 1). Among these 91 patients with non-KD diagnoses, their diagnoses were viral fever, viral conjunctivitis, viral exanthem, measles and upper respiratory tract infection.

In our study, $69.7 \%$ of infants with KD were found to have erythema and induration of the BCG inoculation site when compared to $27.8 \%$ of older children with KD ( $p<0.001$; Table III). In addition, this clinical sign was found to present early in $53.3 \%$ of patients with KD in the course of febrile illness (i.e. within 1-4 days) when compared to $30.0 \%$ of patients with KD who presented with $\geq 5$ days of fever $(p<0.001)$. There was no significant association between BCG site reaction or induration and ethnicity, gender or the number of clinical features of KD (based on the AHA criteria) present.

Among those checked for BCG site reaction or induration, we found low rates of BCG site reaction or induration in patients with non-KD febrile illnesses (9.9\%) when compared to patients with KD (42.6\%) (Table IV). Among patients with non-KD febrile illnesses, nine were found to have erythema or induration of the BCG inoculation site, of which seven were infants. In this group, three patients were diagnosed with viral conjunctivitis, three patients with viral fever, and the rest were diagnosed with viral exanthem, upper respiratory tract infection and measles.

Erythema and induration of the BCG inoculation site was a significant predictor for KD (Table V). The positive predictive value of BCG reaction or induration for KD was $0.908(95 \%$ confidence interval [Cl] 0.819-0.962) for infants and $0.962(95 \%$ $\mathrm{Cl}$ 0.868-0.995) for older children. Erythema and induration of the BCG inoculation site was also found to be significantly more 
Table I. Comparison of demographics between patients with KD and non-KD febrile illnesses ( $n=661$ ).

\begin{tabular}{|c|c|c|c|c|}
\hline \multirow[t]{2}{*}{ Characteristic } & \multicolumn{3}{|c|}{ No. of patients (\%) } & \multirow{2}{*}{$\begin{array}{l}\text { p-value } \\
\text { (KD vs. } \\
\text { non-KD) }\end{array}$} \\
\hline & Overall & $\begin{array}{l}\text { Patients } \\
\text { with KD }\end{array}$ & $\begin{array}{l}\text { Control } \\
\text { patients* }\end{array}$ & \\
\hline \multicolumn{5}{|l|}{ Total $(n=661)$} \\
\hline \multicolumn{5}{|l|}{ Age (yr) } \\
\hline Mean $\pm S D$ & $2.2 \pm 2.4$ & $1.6 \pm 1.8$ & $3.2 \pm 2.8$ & \\
\hline $\begin{array}{l}\text { Median } \\
(I Q R)\end{array}$ & $\begin{array}{l}1 \\
(0-3)\end{array}$ & $\begin{array}{l}1 \\
(0-2)\end{array}$ & $\begin{array}{l}2 \\
(1-4)\end{array}$ & \\
\hline \multicolumn{5}{|l|}{ Gender } \\
\hline Female & $247(37.4)$ & $160(38.1)$ & $87(36.1)$ & \\
\hline Male & $414(62.6)$ & 260 (61.9) & $154(63.9)$ & \\
\hline \multicolumn{5}{|l|}{ Ethnicity } \\
\hline Chinese & $511(77.3)$ & $340(81.0)$ & $171(71.0)$ & \\
\hline Malay & $81(12.3)$ & $42(10.0)$ & $39(16.2)$ & \\
\hline Indian & $21(3.2)$ & $9(2.1)$ & $12(5.0)$ & \\
\hline Other & $48(7.3)$ & $29(6.9)$ & $19(7.9)$ & \\
\hline \multicolumn{5}{|c|}{ Infants ( $n=176)$} \\
\hline Age (yr) & & & & $<0.0001$ \\
\hline Mean $\pm S D$ & $0.005 \pm 0.05$ & $0 \pm 0$ & $0.02 \pm 0.10$ & \\
\hline $\begin{array}{l}\text { Median } \\
(I Q R)\end{array}$ & $\begin{array}{l}0 \\
(0-0)\end{array}$ & $\begin{array}{l}0 \\
(0-0)\end{array}$ & $\begin{array}{l}0 \\
(0-0)\end{array}$ & \\
\hline Gender & & & & 0.698 \\
\hline Female & $58(33.0)$ & $47(33.8)$ & $11(29.7)$ & \\
\hline Male & $118(67.0)$ & $92(66.2)$ & $26(70.3)$ & \\
\hline Ethnicity & & & & 0.010 \\
\hline Chinese & $145(82.4)$ & $120(86.3)$ & $25(67.6)$ & \\
\hline Malay & $19(10.8)$ & $10(7.2)$ & $9(24.3)$ & \\
\hline Indian & $0(0)$ & $0(0)$ & $0(0)$ & \\
\hline Other & $12(6.8)$ & $9(6.5)$ & $3(8.1)$ & \\
\hline \multicolumn{5}{|c|}{ Older children ( $n=485$ ) } \\
\hline Age (yr) & & & & $<0.0001$ \\
\hline Mean $\pm S D$ & $3.0 \pm 2.3$ & $2.4 \pm 1.7$ & $3.8 \pm 2.7$ & \\
\hline $\begin{array}{l}\text { Median } \\
(I Q R)\end{array}$ & $\begin{array}{l}2 \\
(1-4)\end{array}$ & $\begin{array}{l}2 \\
(1-3)\end{array}$ & $\begin{array}{l}3 \\
(2-5)\end{array}$ & \\
\hline Gender & & & & 0.572 \\
\hline Female & $189(39.0)$ & $113(40.2)$ & $76(37.3)$ & \\
\hline Male & $296(61.0)$ & $168(59.8)$ & $128(62.7)$ & \\
\hline Ethnicity & & & & 0.283 \\
\hline Chinese & $366(75.5)$ & $220(78.3)$ & $146(71.6)$ & \\
\hline Malay & $62(12.8)$ & $32(11.4)$ & 30 (14.7) & \\
\hline Indian & $21(4.3)$ & $9(3.2)$ & $12(5.9)$ & \\
\hline Other & $36(7.4)$ & $20(7.1)$ & $16(7.8)$ & \\
\hline
\end{tabular}

*Patients with non-KD febrile illnesses. IQR: interquartile range; KD: Kawasaki disease; SD: standard deviation

specific for KD in older children than in infants, with specificities of 0.971 (95\% Cl 0.900-0.997) and 0.682 (95\% Cl 0.451-0.864), respectively.

\section{DISCUSSION}

Among patients with $\mathrm{KD}$, erythema and induration of the BCG inoculation site has been well reported as a common finding in
Table II. Checking rates of erythema and induration of the BCG inoculation site.

\begin{tabular}{|lll|}
\hline Variable & \multicolumn{2}{c|}{ No. (\%) } \\
\cline { 2 - 3 } & $\begin{array}{l}\text { Infants } \\
\text { (n= 176) }\end{array}$ & $\begin{array}{l}\text { Older children } \\
(\mathbf{n}=\mathbf{4 8 5})\end{array}$ \\
\hline First children's emergency visit & $62(35.2)$ & $150(30.9)$ \\
\hline All children's emergency visits & $81(46.0)$ & $166(34.2)$ \\
\hline Ward & $121(68.8)$ & $249(51.3)$ \\
\hline
\end{tabular}

BCG: Bacillus Calmette-Guérin

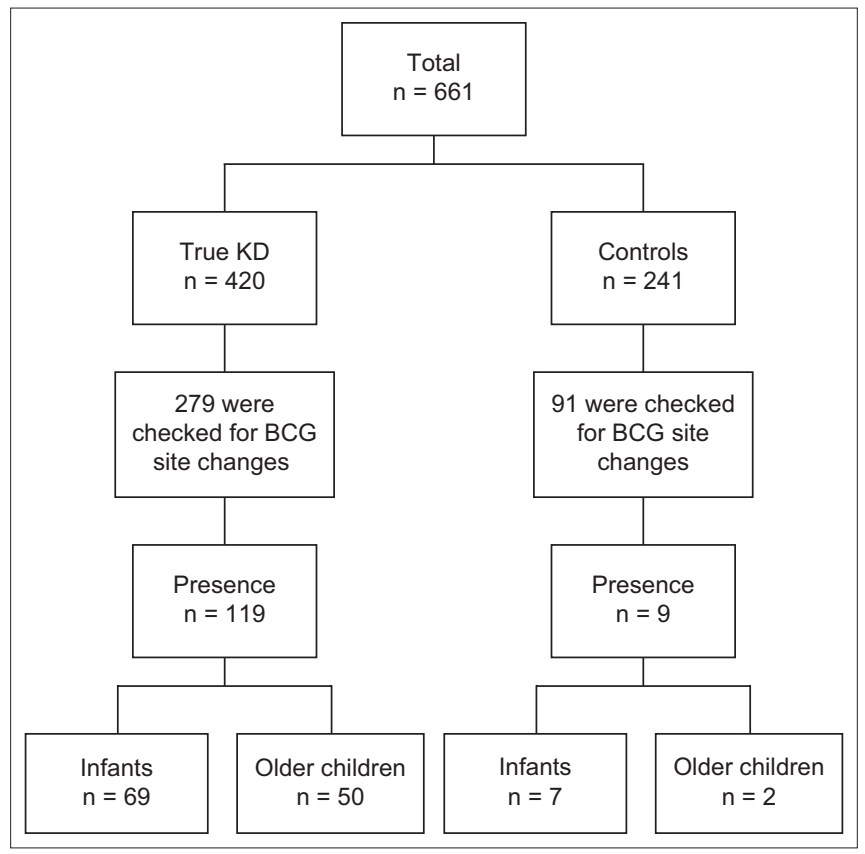

Fig. 1 Flowchart shows analysis of patients with KD versus control patients with non-KD febrile illnesses. BCG: Bacillus Calmette-Guérin; KD: Kawasaki disease

countries with a national BCG vaccination programme, especially for the infant population. Our study reiterated that BCG reaction or induration is more common among infants with $\mathrm{KD}(69.7 \%)$. It was also more prevalent than other clinical features, such as cervical lymphadenopathy or changes in the extremities in the infant population. These findings are consistent with those of the study by Uehara et al in 2010 in Japan, ${ }^{(12)}$ which found erythema and induration of the BCG inoculation site in $70 \%$ of patients with KD aged 3-20 months. They also reported that among patients aged two years or less with complete KD, redness or crust formation at the BCG inoculation site was more prevalent than cervical lymphadenopathy.

We found the BCG site reaction or induration to be more commonly observed 1-4 days after the onset of fever in patients with $\mathrm{KD}$. This relationship between the day of disease onset and appearance of erythema and induration of the BCG inoculation site was also previously reported by Uehara et $\mathrm{al}^{(12)}$ and Hsu et al. ${ }^{(13)}$

Significantly, we observed low rates of BCG site reaction or induration among patients with non-KD febrile illnesses. Of the 128 patients found to have erythema and induration of the BCG inoculation site, nine had a final diagnosis that was not KD 
Table III. Epidemiological characteristics of patients with KD with erythema and induration of the BCG inoculation site in the ward ( $n=279$ ).

\begin{tabular}{|lll|}
\hline Characteristic & \multicolumn{2}{c}{ No. of patients (\%) } \\
\cline { 2 - 3 } & $\begin{array}{l}\text { No erythema and induration } \\
\text { of the BCG inoculation site }\end{array}$ & $\begin{array}{l}\text { Erythema and induration } \\
\text { of the BCG inoculation site }\end{array}$ \\
\hline Age & & \\
\hline Infants $(n=99)$ & $30(30.3)$ & $69(69.7)$ \\
\hline Older children $(n=180)$ & $130(72.2)$ & $50(27.8)$ \\
\hline Gender & & \\
\hline Female $(n=107)$ & $64(59.8)$ & $43(40.2)$ \\
\hline Male $(n=172)$ & $96(55.8)$ & $76(44.2)$ \\
\hline Ethnicity & & $104(45.0)$ \\
\hline Chinese $(n=231)$ & $127(55.0)$ & $8(28.6)$ \\
\hline Malay $(n=28)$ & $20(71.4)$ & $1(16.7)$ \\
\hline Indian $(n=6)$ & $5(83.3)$ & $6(42.9)$ \\
\hline Other $(n=14)$ & $8(57.1)$ & $81(53.3)$ \\
\hline Duration of fever & & $38(29.9)$ \\
\hline$<5$ days $(n=152)$ & $71(46.7)$ & \\
\hline$\geq 5$ days $(n=127)$ & $89(70.1)$ & $52(41.6)$ \\
\hline Clinical features of KD (AHA criteria) & & $67(43.5)$ \\
\hline$\geq 4$ features $(n=125)$ & $73(58.4)$ & \\
\hline$<4$ features $(n=154)$ & $87(56.5)$ & 0.511 \\
\hline
\end{tabular}

AHA: American Heart Association; BCG: Bacillus Calmette-Guérin; KD: Kawasaki disease

Table IV. Diagnoses of patients with erythema and induration of the BCG inoculation site $(n=128)$.

\begin{tabular}{|lll|}
\hline Diagnosis & Final diagnosis in the ward & $\begin{array}{l}\text { No. of } \\
\text { patients }\end{array}$ \\
\hline KD $(n=119 ;$ & KD & 91 \\
\cline { 2 - 3 } $\left.42.6 \%{ }^{*}\right)$ & Atypical/incomplete KD & 28 \\
\hline Non-KD $\left(n=9 ; 9.9 \%^{+}\right)$ & Viral fever & 3 \\
\cline { 2 - 3 } & Viral conjunctivitis & 3 \\
\cline { 2 - 3 } & Viral exanthem & 1 \\
\cline { 2 - 3 } & Measles & 1 \\
\cline { 2 - 3 } & Upper respiratory tract infection & 1 \\
\hline
\end{tabular}

*119 out of total 279 patients with KD who were checked for BCG site erythema or inoculation. +9 out of total 91 control patients with non-KD febrile illnesses; BCG: Bacillus Calmette-Guérin; KD: Kawasaki disease

(control group: 9.9\%; patients with KD: 42.6\%). Among previous studies on febrile illnesses that were not KD, we found only one case report that described an 11 -month-old infant with erythema of the BCG inoculation site due to infection with human herpes virus $6 .^{(14)}$ It has been hypothesised that the T-cell system has a significant role in facilitating vascular endothelial cell injury in KD. ${ }^{(15-17)}$ A recent Taiwanese study in 2016 has attempted to better characterise these changes via dermatoscopy, ${ }^{(11)}$ but further prospective studies of BCG site reactions (either quantified by dermatoscopy or histopathological examination) in patients with KD and other febrile illnesses would be useful to further evaluate this association and its significance.

Of note, seven out of nine patients with non-KD febrile illnesses with BCG site reaction or induration were infants. This paralleled our finding of erythema and induration of the BCG inoculation site to have a higher specificity in older children than
Table V. Diagnostic utility of erythema and induration of the BCG inoculation site in KD in the ward.

\begin{tabular}{|lll|}
\hline Variable & \multicolumn{2}{c|}{ Estimate $(\mathbf{9 5 \%}$ Cl) } \\
\cline { 2 - 3 } & $\begin{array}{l}\text { Infants } \\
(\mathbf{n}=\mathbf{1 2 1})\end{array}$ & $\begin{array}{l}\text { Older children } \\
(\mathbf{n}=\mathbf{2 4 9})\end{array}$ \\
\hline Sensitivity & 0.697 & 0.278 \\
& $(0.597-0.785)$ & $(0.214-0.350)$ \\
\hline Specificity & 0.682 & 0.971 \\
& $(0.451-0.864)$ & $(0.900-0.997)$ \\
\hline Positive predictive & 0.908 & 0.962 \\
value & $(0.819-0.962)$ & $(0.868-0.995)$ \\
\hline Negative predictive & 0.333 & 0.340 \\
value & $(0.200-0.490)$ & $(0.274-0.411)$ \\
\hline
\end{tabular}

BCG: Bacillus Calmette-Guérin; Cl: confidence interval; KD: Kawasaki disease

infants. It is possible that older children possess a more exuberant response to vasculitis due to a more developed immune system, rendering an older child less likely to have KD in the absence of erythema and induration of the BCG inoculation site.

Despite a lower specificity of erythema and induration of the BCG inoculation site in infants, there remains a high positive predictive value for erythema and induration of the BCG inoculation site in this age group. This was consistent with the findings of previous studies by Uehara et $\mathrm{al}^{(12)}$ and Hsieh et al. ${ }^{(18)}$

Notably, in our study, not all physicians documented the presence or absence of this BCG reaction or induration and we were unable to identify any preponderance for certain patients for comparison with others. In our study, among 176 infants who were identified, $121(68.8 \%)$ patients were checked for the presence of erythema and induration of the BCG inoculation 
site. Among the 485 older children identified, 249 (51.3\%) patients were checked for this feature. Checking rates also differed for patients with KD and non-KD diagnoses; $66.4 \%$ of patients with KD and $37.8 \%$ of patients with non-KD febrile illnesses were checked for BCG site changes. Only those who were checked for it were included for further analysis to identify associated epidemiological features. This might have affected the high positive predictive values and relatively low negative predictive values found in our study. Nevertheless, this selective population may be a representative one, as our findings of the prevalence of BCG site reaction or induration compared well with the other $\mathrm{AHA}$ features of $\mathrm{KD}$ and the epidemiological associations of BCG site changes in KD were consistent with other pre-existing studies. Furthermore, suboptimal rates of looking for the presence or absence of BCG site changes reminds us of the fact that vigilance in looking out for this feature should be improved on.

In conclusion, our study highlighted erythema and induration of the BCG inoculation site as a specific clinical sign for KD, especially among children aged one year and above. We also illustrated low rates of BCG changes in febrile illnesses that were not $\mathrm{KD}$. This reinforces the need to be vigilant when looking for erythema and/or induration at the BCG inoculation site in patients presenting with fever. A prompt diagnosis of KD will permit timely intervention, reducing the time-sensitive risk of coronary sequelae in these patients.

\section{ACKNOWLEDGEMENTS}

We would like to thank Li Wenyun, Statistician, Duke-NUS Medical School, Singapore, for her contribution to data analysis. We are also grateful to Adj A/Prof Tan Teng Hong, Senior Consultant, KK Women's and Children's Hospital Cardiology Service, Singapore, for his support of this project.

\section{REFERENCES}

1. Uehara R, Belay ED. Epidemiology of Kawasaki disease in Asia, Europe, and the United States. J Epidemiol 2012; 22:79-85.

2. Golshevsky D, Cheung M, Burgner D. Kawasaki disease--the importance of prompt recognition and early referral. Aust Fam Physician 2013; 42:473-6.

3. Kato $\mathrm{H}$, Sugimura $\mathrm{T}$, Akagi $\mathrm{T}$, et al. Long-term consequences of Kawasaki disease. A 10- to 21-year follow-up study of 594 patients. Circulation 1996; 94:1379-85.

4. Dajani A, Taubert K, Gerber MA, et al. Diagnosis and therapy of Kawasaki disease in children. Circulation 1993; 87:1776-180.

5. Newburger J, Takahashi M, Gerber MA, et al; Committee on Rheumatic Fever, Endocarditis, and Kawasaki Disease, Council on Cardiovascular Disease in the Young, American Heart Association. Diagnosis, treatment, and long-term management of Kawasaki disease: a statement for health professionals from the Committee on Rheumatic Fever, Endocarditis, and Kawasaki Disease, Council on Cardiovascular Disease in the Young, American Heart Association. Pediatrics 2004; 114:1708-33.

6. Ayusawa M, Sonobe T, Uemura S, et al; Kawasaki Disease Research Committee. Revision of diagnostic guidelines for Kawasaki disease (the 5 th revised edition). Pediatr Int 2005; 47:232-4.

7. Takayama J, Yanase Y, Kawasaki T. A study of the cutaneous changes at BCG inoculated sites in MCLS. Pediatr Int 1980; 24:410.

8. Rezai M, Shahmohammadi S. Erythema at BCG inoculation site in Kawasaki disease patients. Mater Sociomed 2014; 26:256-60.

9. Sinha R, Balakumar T. BCG reactivation: a useful diagnostic tool even for incomplete Kawasaki disease. Arch Dis Child 2005; 90:891.

10. Chalmers D, Corban JG, Moore PP. BCG site inflammation: a useful diagnostic sign in incomplete Kawasaki disease. J Paediatr Child Health 2008; 44:525-6.

11. Tseng HC, Ho JC, Guo MM, et al. Bull's eye dermatoscopy pattern at bacillus Calmette-Guérin inoculation site correlates with systemic involvements in patients with Kawasaki disease. J Dermatol 2016; 43:1044-50.

12. Uehara $R$, Igarashi $H$, Yashiro $M$, Nakamura $Y$, Yanagawa $H$. Kawasaki disease patients with redness or crust formation at the Bacille Calmette-Guérin inoculation site. Pediatr Infect Dis J 2010; 29:430-3.

13. Hsu YH, Wang YH, Hsu WY, Lee YP. Kawasaki disease characterized by erythema and induration at the Bacillus Calmette-Guérin and purified protein derivative inoculation sites. Pediatr Infect Dis J 1987; 6:576-8.

14. Kakisaka Y, Ohara T, Katayama S, et al. Human herpes virus type 6 can cause skin lesions at the BCG inoculation site similar to Kawasaki Disease. Tohoku J Exp Med 2012; 228:351-3.

15. Onouchi Y, Gunji T, Burns JC, et al. ITPKC functional polymorphism associated with Kawasaki disease susceptibility and formation of coronary artery aneurysms. Nat Genet 2008; 40:35-42.

16. Kuo HC, Chang WC. Genetic polymorphisms in Kawasaki disease. Acta Pharmacol Sin 2011; 32:1193-8.

17. Lee YC, Kuo HC, Chang JS, et al. Two new susceptibility loci for Kawasaki disease identified through genome-wide association analysis. Nat Genet 2012; 44:522-5.

18. Hsieh KS, Wang CF, Wong KP, Lin CC. Erythema at a BCG inoculation site in Kawasaki disease with coronary artery ectasia. Poster Program. Pediatr Int 2012; 54:20-35. 